\title{
Fate of large-scale vortices in idealized tidal lagoons
}

\author{
Carolanne V. M. Vouriot ${ }^{1}$ - Athanasios Angeloudis ${ }^{2}$ (D) - Stephan C. Kramer ${ }^{2}$. \\ Matthew D. Piggott ${ }^{2}$
}

Received: 19 March 2018 / Accepted: 4 September 2018 / Published online: 19 September 2018

(C) The Author(s) 2018

\begin{abstract}
The generation and evolution of tidally-induced vortices in coastal and estuarine regions can influence water quality and sedimentary processes. These effects must be taken into consideration in the development of coastal reservoirs, barrages and lagoons, among other environmental flow applications. Results are presented here on the fate of large-scale vortices within confined tidally-forced domains. A computational approach is employed using the Thetis depth-averaged coastal ocean modeling framework. Initially, two test cases serve to demonstrate model capability in capturing the formation of dipoles downstream of oscillatory flow channels. Diagnostic quantities of vorticity and localized circulation are used to track the 2-D vortex evolution and dissipation. This approach is then applied to tidal lagoon geometries, where flows through the inlet induce a pair of counter rotating vortices (dipoles). Idealized model geometries and inlet conditions are used to determine the impact of three design parameters on large-scale vortical structures: (a) the lagoon geometry aspect ratio in the horizontal plane, (b) the inlet width and (c) the bathymetry profile as the coastline is approached. The dependence of vortex flushing behavior on the dimensionless ratio ${ }^{W_{\mathrm{i}}} / U T$ (where $W_{\mathrm{i}}$ is the width of the inlet channel, $U$ is the maximum velocity and $T$ is the tidal period) is reaffirmed, while the side walls and the sloping bathymetry are found to affect the vortex dissipation process.
\end{abstract}

Keywords Tidal lagoons $\cdot$ Vortices $\cdot$ Tidal modeling $\cdot$ Environmental impacts

Athanasios Angeloudis

a.angeloudis06@imperial.ac.uk

Carolanne V. M. Vouriot

carolanne.vouriot12@imperial.ac.uk

1 Fluid Mechanics Section, Department of Civil and Environmental Engineering, Imperial College, London, UK

2 Applied Modelling and Computation Group, Department of Earth Science and Engineering, Imperial College, London, UK 


\section{Introduction}

The presence of tidally-induced vortices in coastal flows is a common phenomenon. Vortices tend to form when tidal currents encounter particular geometries, such as when flowing around sharp headlands [1,2] or islands [3-5], and within confined estuaries [6], coastal reservoirs or harbors [7]. These vortices can become hazardous for navigation and affect the entrainment and mixing of sand (potentially creating sandbanks), pollutants or nutrients [8]. The formation of large-scale two-dimensional vortices was recently observed [9] downstream of what is currently the world's largest tidal range based power generation scheme in Lake Sihwa, South Korea. In that case, patterns were identified between the occurrence of recirculation zones and the local suspended sediment transport regime. Given how coastal reservoirs and marine energy developments including tidal lagoons are gaining interest [10-12], it is timely to consider the fate of such vortices in the context of confined tidal embayments.

The formation of vortices through tidal inlet flows has been investigated analytically, experimentally and computationally, e.g. [13-16]. As an example, Wells and Heijst [13] applied potential theory by representing the dipoles as a source-sink system to determine whether the vortices will be long-lived (i.e. surviving longer than a tidal cycle and gradually dissipating downstream from the tidal inlet), or whether they would return towards the inlet as the tide recedes and be eventually flushed out from the impounded area with the outgoing flow. This behavior can be examined by considering the ratio of the amount of water that escapes the influence of the sink to the amount of water that enters with the flood tide. This has been found to be dependent on the inlet flow propagation velocity, which needs to be sufficiently high for the vortices to escape from the sink flow during the ebb tide. Experimental and analytical findings show vortices escape from the inlet provided $W_{\mathrm{i}} / U T$ is smaller than a critical value of $\sim 0.13$ [13] (where $W_{\mathrm{i}}$ is the width of the inlet channel, $U$ the maximum velocity and $T$ the tidal period). Subsequently, experimental results on the propagation of tidally-induced vortices were reported using tracer dye injections and particle image velocimetry (PIV) that were applied to a series of physical modeling campaigns of tidal inlet geometries $[14,16]$.

In the assessment of coastal reservoirs and lagoons, computational approaches have been reported that approximate the problem as anything from a 0-D [17], 1-D [18], 2-D [19-21] and 3-D [22] system. Due to the wide range of scales present in such engineering applications, hydrodynamic modeling that is capable of resolving the formation of near-field recirculation zones is feasible in only a subset of these approaches. Two-dimensional (2-D) depth-averaged modeling is often selected as it strikes a balance between accuracy and computational complexity, and allows for the consideration of certain regional and far-field effects. The presence and potential implications of large recirculation zones has been discussed in several studies [23]. For example, low-velocity flow areas at the zones' centers can promote sediment accumulation through the tealeaf paradox [24].

In this paper we consider flow within idealized tidal lagoon geometries with a focus on the generation and propagation of two-dimensional vortex hydrodynamic structures. A computational approach is employed utilizing an unstructured mesh coastal ocean model. This is motivated in part by the need to deliver an impact assessment capability that can be transferred to more realistic representations of coastal engineering infrastructure in shallow water flows. Two scenarios are initially considered to validate the model setup and demonstrate its ability to characterize the primary vortex transport. 
Sequentially, simplified lagoon designs and inflow conditions are investigated to identify key parameters for the generation of long-enduring vortices.

\section{Methodology}

We consider flow simulations through tidal inlets of confined embayments performed using Thetis [25, 27], a coastal ocean modeling framework implemented using the Firedrake finite element partial differential equation (PDE) solver engine [26]. Thetis features 3-D modeling capabilities [27] and has already been applied for the simulation and assessment of tidal range structure operation strategies [28]. The configuration of the model presented here is depthaveraged, assuming homogeneity in the vertical direction, and solves the non-conservative form of the 2-D shallow water equations:

$$
\begin{gathered}
\frac{\partial \eta}{\partial t}+\nabla \cdot\left(H_{d} \mathbf{u}\right)=0, \\
\frac{\partial \mathbf{u}}{\partial t}+\mathbf{u} \cdot \nabla \mathbf{u}-v \nabla^{2} \mathbf{u}+g \nabla \eta=-\frac{\tau_{b}}{\rho H_{d}},
\end{gathered}
$$

where $\eta$ is the free surface perturbation, $H_{d}=\eta+h_{m}$ is the total water depth with $h_{m}$ being the mean water depth and $\mathbf{u}$ is the depth-averaged velocity vector with horizontal components $u, v$ in the $x, y$ directions respectively. $v$ represents kinematic viscosity in the model which is typically equal to the aggregate value of molecular and turbulent viscosities. The fluid molecular viscosity is considered negligible and a constant eddy-viscosity turbulence model is applied for simplicity in this work. The bed shear stress can be represented by the quadratic drag coefficient $C_{D}$ as follows [25]:

$$
\frac{\tau_{b}}{\rho}=-C_{D}|\mathbf{u}| \mathbf{u}
$$

or by imposing Manning's formulation as

$$
\frac{\tau_{b}}{\rho}=g n^{2} \frac{|\mathbf{u}| \mathbf{u}}{H_{d}^{\frac{1}{3}}},
$$

with $n$ the Manning's coefficient $\left(\mathrm{s} \mathrm{m}^{-1 / 3}\right)$. Intertidal areas constitute a characteristic of most tidal estuaries with wetting and drying processes being challenging to represent numerically [29]. Thetis employs the formulation described in Kärnä et al. [30] that uses a negative depth approach where the bathymetry is allowed to move artificially conserving continuity while also being applicable to tracers. The model is implemented using a discontinuous Galerkin finite element discretization (DG-FEM), using the $\mathrm{P}_{1 \mathrm{DG}}-\mathrm{P}_{1 \mathrm{DG}}$ velocity-pressure finite element pair. A semi-implicit Crank-Nicolson timestepping approach is applied for temporal discretization with a constant timestep of $\Delta t$. The discretized equations are solved by a Newton nonlinear solver algorithm through the PETSc library [31].

Vorticity, defined in the strong form as $\omega=-\frac{\partial u}{\partial y}+\frac{\partial v}{\partial x}$ is given in the weak form using a test function $\psi$ as:

$$
\int_{\Omega} \psi \omega \mathrm{d} \Omega=\int_{\Omega} \psi\left[-\frac{\partial u}{\partial y}+\frac{\partial v}{\partial x}\right] \mathrm{d} \Omega
$$


where $\Omega$ is the simulation domain. Integrating by parts and applying the Gauss divergence theorem, this expands as:

$$
\begin{aligned}
\int_{\Omega} \psi \omega \mathrm{d} \Omega & =\int_{\Omega} \frac{\partial v}{\partial x} \psi \mathrm{d} \Omega-\int_{\Omega} \frac{\partial u}{\partial y} \psi \mathrm{d} \Omega \\
& =\int_{\Gamma} v \psi \mathrm{d} s-\int_{\Omega} v \frac{\partial \psi}{\partial x} \mathrm{~d} \Omega-\int_{\Gamma} u \psi \mathrm{d} s+\int_{\Omega} u \frac{\partial \psi}{\partial y} \mathrm{~d} \Omega \\
& =\int_{\Omega}\left(-u \frac{\partial \psi}{\partial y}+v \frac{\partial \psi}{\partial x}\right) \mathrm{d} \Omega+\int_{\Gamma}\left(\psi u n_{y}-\psi v n_{x}\right) \mathrm{d} \Omega,
\end{aligned}
$$

where $\Gamma$ represents the boundary of our domain, $d s$ the boundary integral and $n_{x}, n_{y}$ the exterior facets at the boundaries in the $x$ and $y$ directions respectively. A piecewise linear, continuous Galerkin finite element approximation of vorticity can now be found by solving the system of equations formed by (6) and a basis of test functions $\psi$ in the space of piecewise linear functions, for a function $\omega(x)$ in that same function space.

In order to track vortices during the simulations we first identify areas of high $\omega$. For each of these areas, the circulation (i.e. the velocity line integral or effectively the flux of $\omega)$, denoted here $\Gamma_{c}$, is calculated as:

$$
\Gamma_{c}=\iint_{S} \omega d S,
$$

where $S$ is a closed surface around the vorticity peaks, with the area being dependent on the scale of the problem. Once a peak in the $\omega$ field is identified, $\omega$ is integrated over a circular area $S$ centered at the peak with a radius $r$ (7). The value of the radius $r$ effectively dictates the size of the vortices that will be tracked and was selected according to the size of the large scale vortices that are examined. For the simulation domains considered in Sects. 3.2 and 4 a value of $r=500 \mathrm{~m}$ was applied. Recirculation zone centers were distinguished from shear-induced $\omega$ peaks through a threshold value $\left(\left|\Gamma_{\text {critical }}\right|\right)$ that aims to exclude peaks that feature a high $\omega$ value at the core but a low value around it; such a combination renders these points unlikely to correspond to vortex cores. Determining this value was the matter of a preliminary sensitivity assessment with $\left|\Gamma_{\text {critical }}\right| \propto r / h_{l}$ where $h_{l}$ is a representative element length over the area $S$ to account for resolution differences. The algorithm evaluates the proximity of each peak to vortex core coordinates from previous timesteps and either appends the new coordinates to known vortex trajectories or establishes a new trajectory.

\section{Numerical model capabilities, validation and sensitivity}

Hydrodynamic models need to be calibrated and validated to ensure that the physical processes of interest are simulated with sufficient accuracy [32]. This is typically achieved for regional-scale simulations through comparisons against observed tidal constituents and field measurements such as water elevation records (e.g. tide gauge data) or velocity samples (Acoustic Doppler Current Profiler readings). Depth-averaged numerical modeling methods have proven their accuracy in many coastal modeling applications if the assumptions underlying the shallow water equations remain valid [23, 33]. In particular, as long 
as the coastal flow can be assumed to be independent of depth, i.e. possessing minimal three-dimensionality, depth-averaged models can efficiently and practically simulate water elevations over extended periods of time. Nonetheless, once extending the applications to marine energy and in the context of tidal barrages, certain 2-D modeling limitations have been observed nearfield to the hydraulic structures (turbines and sluice gates) where the flow becomes strongly three-dimensional and a 2-D model is thus expected to fail in capturing the true dynamics [24]. Therefore, computationally demanding modeling approaches become essential if 3-D profiles such as the flow immediately adjacent to the hydraulic structures are to be characterized [18, 34, 35]. According to physical modeling experiments [22], the predominantly 3-D flow regions are typically contained within a distance of $20 \mathrm{D}$ (where $D$ is the turbine diameter). These near-field scales are beyond the scope and capabilities of the regional-scale models considered here.

Limitations of 2-D approaches must be acknowledged in the context of vortex modeling. Tide-induced vortices can feature 3-D behavior due to vertical secondary flows. These lead to the upwelling of water from the bottom boundary layers towards the surface and through the center of the horizontal recirculation [36, 37]; these effects will also contribute to the aforementioned accumulation of sediments towards the core. Another physical mechanism that might be neglected is transverse advection in the vertical direction discussed in earlier studies [38]. Its effects are more pronounced in regions where stratification is dominant. For sites that feature a tidal range of interest for marine renewable energy developments, the flows are typically well-mixed and therefore weakly, or not, stratified (e.g. in the Bristol Channel in the UK or the Rance river in France). Nonetheless, given the extended slack water times expected within tidal range structures, internal conditions would potentially merit an expansion to 3-D modeling as stratification effects might emerge. In practice, neglecting such processes could lead to an overestimation of vortex endurance [36] and any associated hydro-environmental implications.

Two test cases (one laboratory scale and one full scale) of oscillatory flow through tidal inlets are considered. Case A examines the capability of Thetis as a means to study the formation of tide-induced vortices where we make comparisons against experimental and numerical findings from Kim et al. [14]. Case B follows Wells and Heijst [39] and serves to determine whether vorticity $(\omega)$ and circulation $\left(\Gamma_{c}\right)$ quantities can be used to diagnose the fate of these vortices. In addition, Case B forms the basis for a grid sensitivity assessment to highlight the dependence of the $\omega$ diagnostic on the localized mesh resolution and its significance for the study findings.

\subsection{Case A: Oscillatory flow through a tidal inlet physical model}

In the study of Kim et al. [14], a series of test cases are presented for the validation of a depth-integrated numerical model based on experimental results. In considering the behavior of oscillatory flow in a shallow water basin containing an inlet structure (denoted as Layout D in [14]), measurements and numerical results were compared. The particular setup is schematically illustrated in Fig. 1a for completeness.

Thetis was configured to replicate the recorded flow conditions beginning from equilibrium with a still water depth of $0.1 \mathrm{~m}$, a friction coefficient $C_{D}=0.0075$, a viscosity of $v=0.001 \mathrm{~m}^{2} \mathrm{~s}^{-1}$, a mesh resolution of $0.02 \mathrm{~m}$ and a time step of $\Delta t=0.1 \mathrm{~s}$. The tidal flow was simulated by imposing oscillatory fluxes at each end of the basin. The fluxes were set to $23 \mathrm{~L} \mathrm{~s}^{-1}$ with a period of $55 \mathrm{~s}$ and a delay of $27.5 \mathrm{~s}$ between the right and left open boundaries. These settings correspond to the time series of width-averaged 
(a)

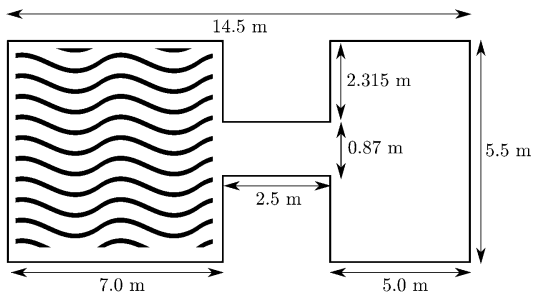

@monitoring domain (b)

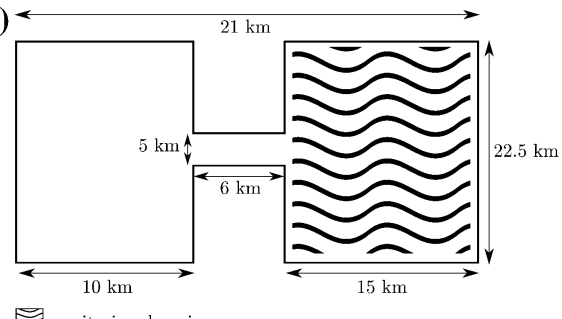

Fig. 1 Layout of the two validation cases considered. a Physical model tidal inlet case as per Kim et al. [14]. b Tidal channel case of Wells and Heijst [39]. The monitoring domain identified by wavy lines indicates the region shown in later plots and diagnostics. The tidal inlet refers to the narrower channel in the center of the domains. For the purposes of illustration only, dimensions not to scale

velocities across the mouth of the tidal inlet shown in Fig. 2, which can be seen to agree well with data reported from the experiment. The vorticity field plotted over the left basin (schematically illustrated in Fig. 1a) is in alignment with the results of Kim et al. [14] in Fig. 3. The exact magnitude and timing of the boundary forcing had to be calibrated to match the velocities through the tidal inlet, with the boundary forcing being multiplied by a ramp function in the form of $r(t)=\tanh \left(\left(t-t_{0}\right) / t_{r}\right)$ where $t_{0}=0$, and $t_{r}=T / 4$ to simulate the time required for the experiment to commence from equilibrium conditions. The $t_{r}$ parameter was determined through preliminary calibration.

Figure 3 demonstrates good performance in terms of capturing the formation and propagation of the counter-rotating vortices. A better agreement is observed in the three latter instantaneous plots of vorticity, as the simulation becomes independent of the initial equilibrium conditions. Minor deviations in the exact location of the core appear which could be attributed to differences in the meshes used between the two studies (such as a structured vs unstructured mesh based approach). However, these results

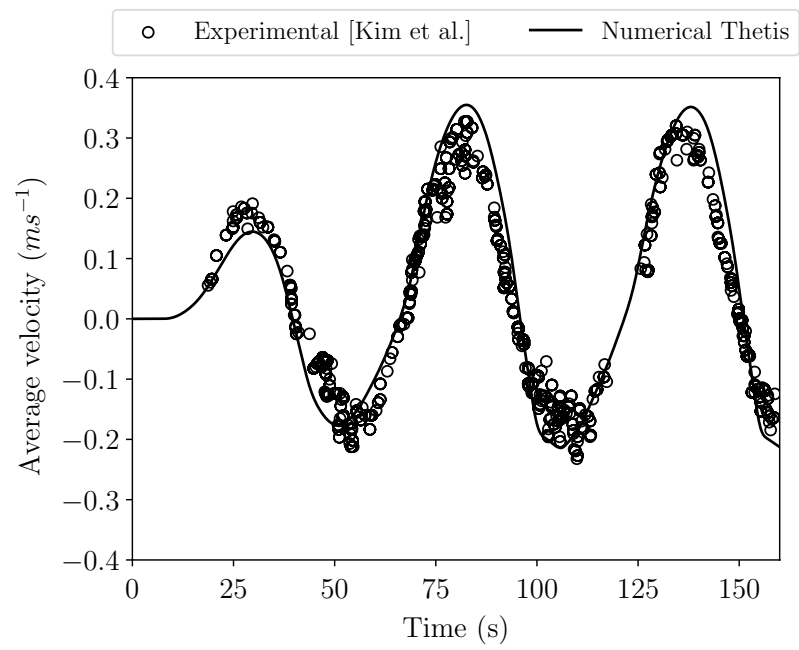

Fig. 2 Comparison of mean velocities at the left hand end of the central channel between the numerical results computed here and the experimental results [14] 
(a) $t=39 \mathrm{~s}$

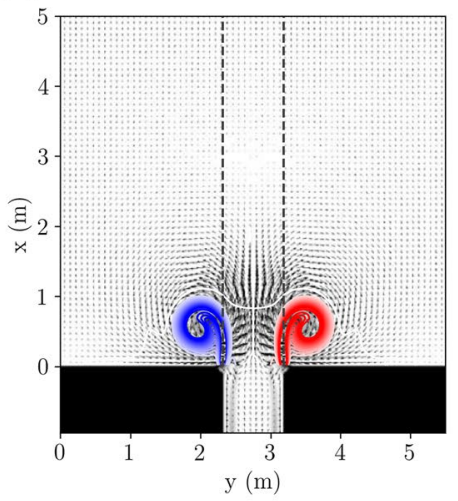

(c) $t=117 \mathrm{~s}$

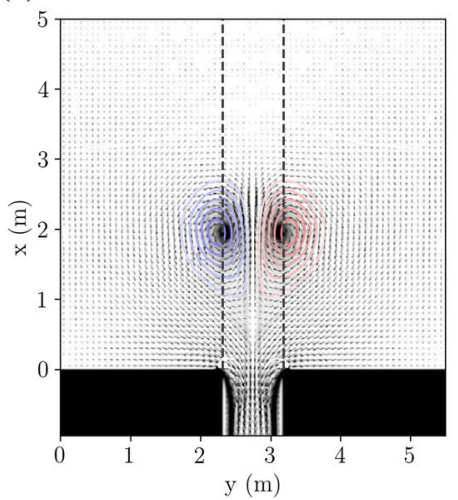

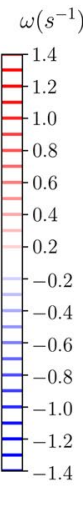

$\omega\left(s^{-1}\right)$

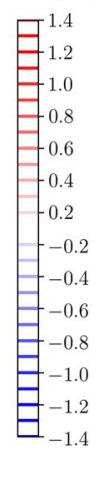

(b) $t=90 \mathrm{~s}$

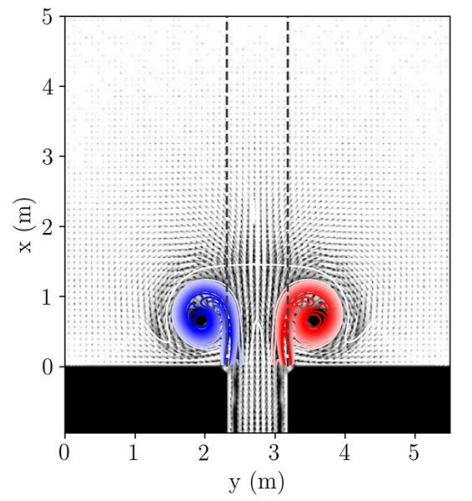

$\omega\left(s^{-1}\right)$

(d) $t=142 \mathrm{~s}$

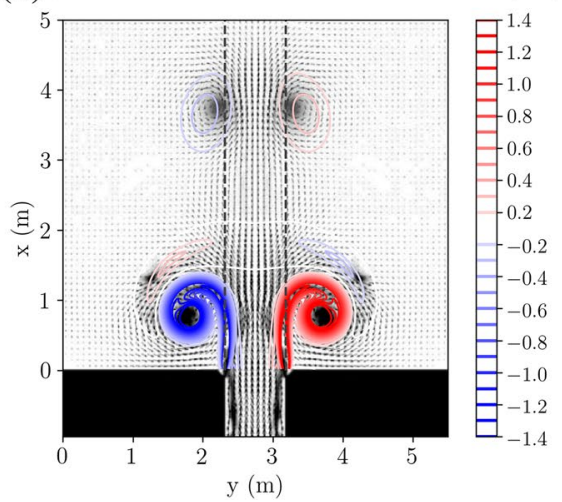

Fig. 3 Qualitative comparison between numerically-predicted vorticity fields of [14] at $\mathbf{a} t=39 \mathrm{~s}, \mathbf{b} t=90$ $\mathrm{s}, \mathbf{c} t=117 \mathrm{~s}$ and $\mathbf{d} t=142 \mathrm{~s}$ and corresponding contour plots from the Thetis simulations

provide us with confidence in the ability of the model to simulate the generation and propagation of vortices.

\subsection{Case B: Dipole formation by tidal flow in a channel}

The second case is based on the investigation of Wells and Heijst [13, 39] into the propagation of vortices formed through idealized tidal inlet flows. Wells and Heijst [13] observed how counter-rotating vortices can form a coherent dipole structure that can self-propagate away from the channel. By treating the dipoles as a source-sink pair, their trajectory was plotted for different $W_{\mathrm{i}} / U T$ values and it was shown that the vortices are expected to escape from the central channel and into the confined basin if $W_{\mathrm{i}} / U T=0.13$.

Numerical simulations consider the domain shown in Fig. $1 \mathrm{~b}$ as per the case study of Wells and Heijst [39]. We initially consider two instances of bottom friction; these are represented through quadratic drag coefficients of (a) $C_{D}=2.5 \times 10^{-3}$ and (b) $C_{D}=1.25 \times 10^{-3}$. In addition, we test three unstructured mesh resolutions with element edge lengths of $h=400,200$ and $100 \mathrm{~m}$ respectively. A structured mesh of $100 \mathrm{~m} \times 100$ $m$ was employed previously [39] based on the justification that this adequately represented 
the bathymetry which takes a maximum value of $20 \mathrm{~m}$ in the domain interior and linearly decreases to $0 \mathrm{~m}$ within a distance of $2.5 \mathrm{~km}$ at all boundaries other than the inlet. Wetting and drying features thus appear as a result.

The tidal elevation was defined at the left hand boundary via a sinusoidal forcing of 1 $\mathrm{m}$ amplitude. All others boundaries, including the inlet channel, were treated with a noslip condition. A tidal period of $T=9 \mathrm{~h}$ was set for the tidal forcing as it led to predictions that reflect the values of the channel velocities reported in Wells and Heijst [39]. Simulations over the two coarser meshes were run with $\Delta t=50 \mathrm{~s}$. On the finer mesh the timestep was reduced to $25 \mathrm{~s}$ to avoid instabilities related to the Courant-Friedrichs-Lewy (CFL) criterion. The simulations were ramped-up over four tidal periods, and the analysis was performed over the subsequent three periods to ensure independence from the initial equilibrium state specified for the model. The $\omega$ field was monitored every $200 \mathrm{~s}$ and processed to track vortices using Eq. 7 and the $\omega$ peaks (Eq. 6) across the domain.

The velocity amplitude is $\approx 1 \mathrm{~m} \mathrm{~s}^{-1}$, corresponding to a ratio of $W_{\mathrm{i}} / U T=0.154$ which suggests that vortices will be eventually flushed out of the confined lagoon by the ebbing flow. However, it was observed [13] that bathymetry and bottom friction effects will be influential on the vortex fate even though these factors are not inherently included in the ratio evaluation. The simulation results of Fig. 4 demonstrate how with a drag coefficient of $C_{D}=2.5 \times 10^{-3}$ the vortices dissipate rapidly in the flushing process, while with the lower $C_{D}=1.25 \times 10^{-3}$ vortices were found to propagate further into the lagoon, enduring longer and dissipating gradually.

Values of the core $\omega$ and coordinates $\left(\omega_{c}, x_{c}, y_{c}\right)$ were tracked in time within the confined area. Figure 4 illustrates the bottom half of the lagoon where the vortex of positive $\omega$ is transported. The equivalent negative $\omega$ vortex propagating within the top half of the monitoring domain is omitted here on the grounds of symmetry. Maximum and minimum values of $\omega$ are shown in Fig. 5 for the three mesh resolutions, plotted together with $\omega_{c}$. The greatest $\omega$ magnitude within the monitoring domain is attributed to shear-induced effects at the inlet corners and on the sides of the incoming flow at flood tide. In order to track vortices, the $\omega$ maxima/minima were therefore generally only monitored in the sub-region $x>16.5 \mathrm{~km}$ (as seen from the starting location of the trajectories plotted in Fig. 4) because this successfully identifies the values at the primary vortex core. However,
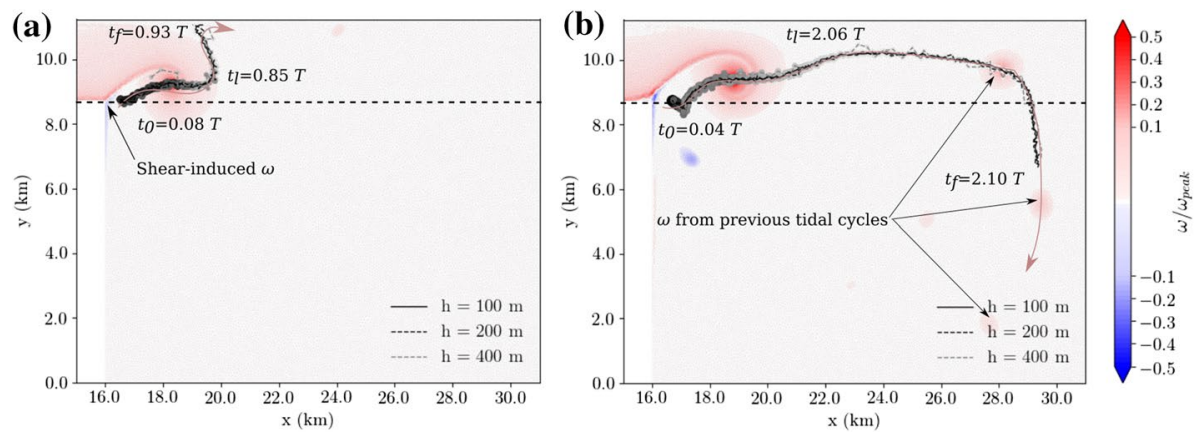

Fig. 4 Vorticity field $19.45 \mathrm{~h}$ after the spin-up period for $\mathbf{a} C_{D}=2.5 \times 10^{-3}$ and $\mathbf{b} C_{D}=1.25 \times 10^{-3} \cdot t_{l}$ here represents the duration of the vortex lifetime while $t_{0}$ and $t_{f}$ mark the appearance and dissipation points of the circulation around the vortex core. The vorticity fields are normalized by the maximum instantaneous vorticity in the $\mathbf{b}$ simulation: $\omega_{\text {peak }}=0.014 \mathrm{~s}^{-1}$. In $\mathbf{b}$ vortex cores remaining from previous tidal cycles are also identified 

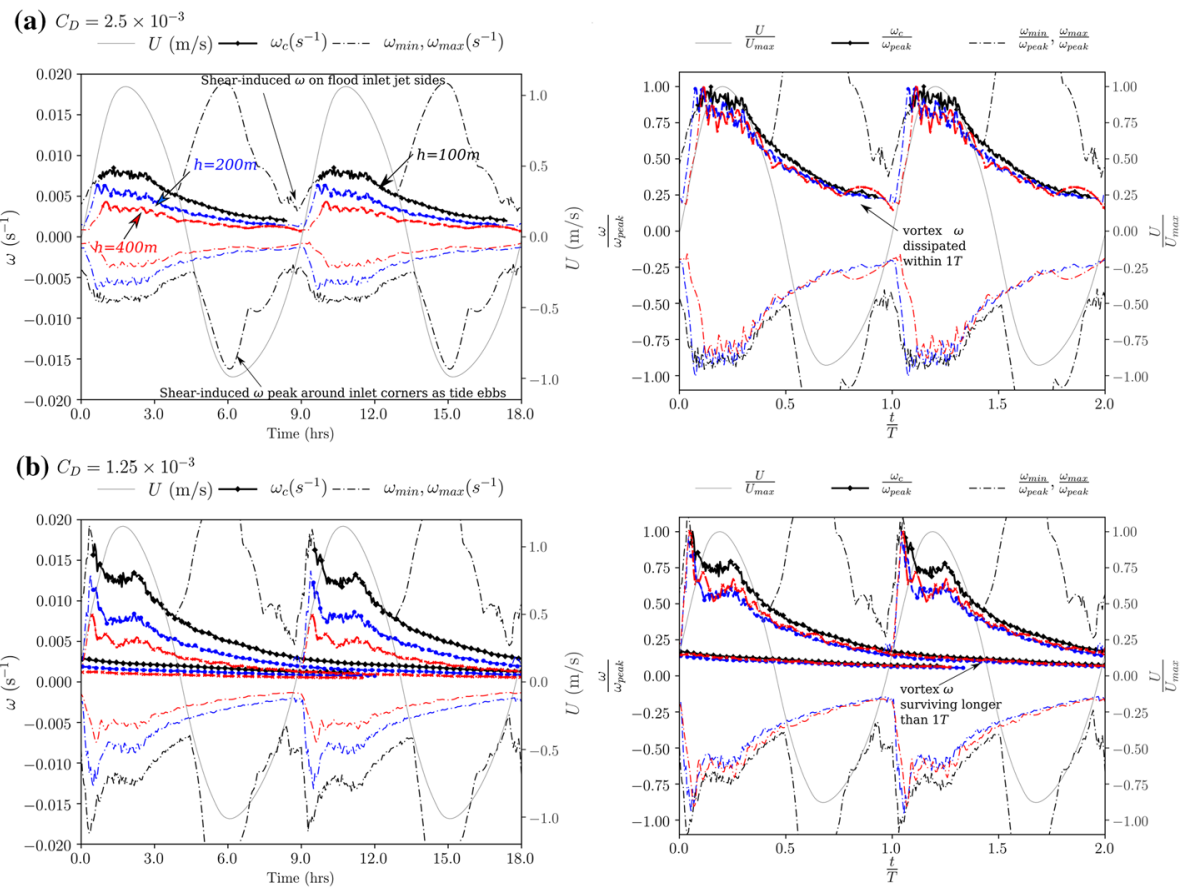

Fig. 5 Maximum $\left(\omega_{\max }\right)$, minimum $\left(\omega_{\min }\right)$ and vortex core $\left(\omega_{\mathrm{c}}\right)$ vorticity values plotted together with the tidal channel velocity against time for $\mathbf{a} C_{D}=2.5 \times 10^{-3}$ and $\mathbf{b} C_{D}=1.25 \times 10^{-3}$. The figure values on the right are normalized with respect to $U, \omega$ and $t$ using the maximum velocity at the inlet $\left(U_{\max }\right)$, the peak vorticity at the core center in each case $\left(\omega_{\text {peak }}\right)$, and the tidal period $(T)$ of the simulations respectively. In the $C_{D}=1.25 \times 10^{-3}$ case evidence of vortices from previous tidal cycles can be seen in the vorticity plots

to demonstrate the scale of the non-recirculating zone $\omega$ immediately adjacent to the inlet, in the $h=100 \mathrm{~m}$ case the maximum values were monitored for $x>16 \mathrm{~km}$ and plotted in Fig. 5. What becomes apparent in Fig. 5 is that for a lower friction coefficient the vortices dissipate significantly later, and can be traced multiple tidal cycles from their origin (see Fig. 4b). Wells and Heijst [13] argued that vortices should endure longer in deeper water if the same tidal inlet velocities are assumed and for $C_{D}=2.5 \times 10^{-3}$. This is to be expected given that in deeper water bed-shear effects should impact the depth-averaged flow profile to a lesser extent (as estimated by the Manning formulation in Eq. 4) and there will be a higher wave celerity $C_{0}=\sqrt{g H_{d}}$. Practically, increasing the maximum depth $\left(H_{\max }\right)$ while maintaining the seaward amplitude $\alpha$ reduces the flow velocity magnitude by distributing the inlet flow over a greater surface area and this drastically increases $W_{\mathrm{i}} / U T$.

A grid sensitivity analysis based on the the element edge lengths $(h)$ serves to assess whether the mesh resolution is sufficient to capture the formation and evolution of vortices. It is crucial to observe that $\omega$ relies on the curl of the $\mathbf{u}$ vector, which in turn requires the spatial gradient of the velocity components as expressed in the weak form in Eq. (6). Unlike the velocity field, a finer mesh resolution corresponds to higher values of $\omega$ as Fig. 5 demonstrates. This is not an unexpected outcome and is often seen in diagnostic quantities involving derivatives. Finer resolutions are capable of representing smaller hydrodynamic structures and therefore higher gradients, and in addition, coarser meshes potentially lead to a higher degree of implicit numerical viscosity. Notwithstanding the foregoing, the 
predictive capability of the model in terms of the vortices being dissipated or not remains consistent for all the resolutions tested, even though a coarser resolution could fail to capture detailed features beyond a certain point.

As we are primarily interested in investigating whether vortices are dissipated over the course of the tidal cycles, $\omega$ was normalized according to the peak values $\left(\omega_{\text {peak }}\right)$ as shown in the right hand side of Fig. 5. This is convenient here as uniform $h$ lengths characterize the domain of the test cases. Once normalized, results from the three mesh resolutions appear to yield very similar trends as to how $\omega$ in the domain gets dissipated. Fluctuations in the $\omega$ values monitored at the core (observed in Fig. 5) are attributed to the tracking algorithm that is based on nodal values rather than the entire field (see Fig. 4); as the resolution increases, these fluctuations decrease in magnitude.

Replicating this test case illustrates the model's capability in capturing the formation and propagation of vortices created by tidal flow. In particular, the reproduction of previous findings [39] serves to demonstrate how the new modeling approach of Thetis agrees with the results of established software (in this case the finite difference Delft3D code). The behavior of the vortices can be monitored though the combination of $\omega$ and $\Gamma_{c}$, with their fate being dependent on parameters such as the bathymetric depth $H$, the inlet velocity $U$, the channel width $W_{\mathrm{i}}$, the tidal period $T$ and bed shear effects (represented for this case study through the quadratic drag coefficient $C_{D}$ ).

\section{Application to idealized tidal lagoons}

This section presents simulation results concerning the behavior of vortices created at the inlet of tidal range structures. Techniques applied in the case studies (A and B) are extended to a set of idealized lagoons. Three parameters are varied when designing the test lagoons: the aspect ratio of the lagoon sides ( $L / W$-domain length/width), the width of the inlet $\left(W_{\mathrm{i}}\right)$ representing the turbine housing, and the bathymetry. The aim is to demonstrate how these affect the fate of the vortices created by the inlet water jets.

Lagoons are modeled as rectangular domains with a flux boundary condition at an opening centered at the middle of the lagoon's left hand side. The opening can be perceived as a representation of the open boundary of turbine caissons [24]. The tidal lagoon simulations do not feature an inlet channel and the flow is evenly distributed across the boundary width assuming a uniform distribution of equally sized hydraulic structures that are driven by head differences, such as turbines or sluice gates. This means that curvature and vorticity quantities produced within a conventional inlet channel are not present in this case. The lagoon geometric specifications are illustrated in Fig. 6 and summarized in Table 1.

Table 1 Lagoon geometry specifications

\begin{tabular}{lllll}
\hline Lagoon & $L(\mathrm{~km})$ & $W(\mathrm{~km})$ & $L / W(-)$ & $\begin{array}{l}\text { Sloping } \\
\text { bathymetry } \\
(\%)\end{array}$ \\
\hline$L_{25 / 4}$ & 12.5 & 2 & 6.25 & 16 \\
$L_{25 / 16}$ & 6.25 & 4 & 1.5625 & 32 \\
$L_{1}$ & 5 & 5 & 1 & 40 \\
$L_{16 / 25}$ & 4 & 6.25 & 0.64 & 50 \\
$L_{4 / 25}$ & 2 & 12.5 & 0.16 & 100 \\
\hline
\end{tabular}




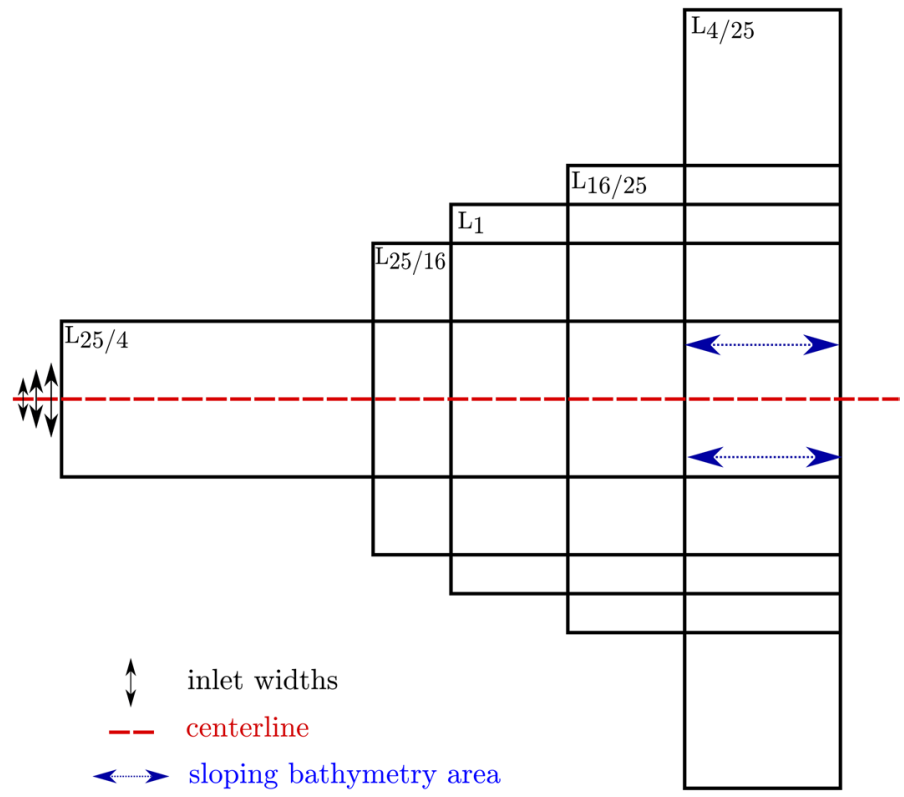

Fig. 6 Diagram illustrating the five lagoons studied

These are defined in a manner that enables assessment of side wall proximity and sloping bathymetry effects, with the right hand wall of the lagoon assumed to coincide with the coastline. Five domain aspect ratios $(L / W)$ were considered, each constrained to yield an overall rectangular area of $25 \mathrm{~km}^{2}$. Consequently, all options theoretically deliver the same power output when subject to identical tidal forcing downstream. As the domain width $(W)$ increases so does the amount of the internal surface area affected by intertidal processes; in practice this will influence the power generation performance. The maximum bathymetry was set to $40 \mathrm{~m}$ sloping up to $0 \mathrm{~m}$ over a distance of $2 \mathrm{~km}$ on the landward (right-hand) boundary of the lagoon. This implies that the widest lagoon $\left(L_{4 / 25}\right)$ was entirely covered by the sloping bathymetry.

Three inlet width cases (of $W_{\mathrm{i}}=1.25,1.00$ and $0.75 \mathrm{~km}$ ) were successively tested for every instance of domain aspect ratio $(L / W)$. For a tidal energy scheme $P \propto A H^{2}$, where $A$ is the impounded surface area and $H$ is the head difference between the inner and outer elevations [40]. As a result, for a given area $A$ and under idealized conditions the same number of turbines would be installed and the remaining control factor is the distribution of the hydraulic structures over the lagoon's seaward side wall. In all simulations the same sinusoidal flux was considered to represent a broader or narrower, yet uniform, distribution of incoming flow over the inlet width. Tidal forcing was applied by specifying the flux at the inlet in the $x$ direction and its period was set to $T=6 \mathrm{~h}$ starting with an ebb tide. Finally, the maximum flux at the inlet was set to $18,000 \mathrm{~m}^{3} \mathrm{~s}^{-1}$ for the width of the inlet to have a significant impact on the vortex transport.

The lagoon domain meshes correspond to an element edge length of $h=50 \mathrm{~m}$ which is consistent with the values used in Sect. 3.2 with respect to the resolution of the inlet geometry. The time step $\Delta t$ of the hydrodynamic simulations was set to $10 \mathrm{~s}$, which satisfies the CFL criterion throughout the duration of the runs. Each case was first ramped up 
over four tidal periods and the vorticity fields were then processed every 200s over the subsequent periods. A viscosity sponge was applied at the inlet where a maximum viscosity of 10 drops to $0.1 \mathrm{~m}^{2} \mathrm{~s}^{-1}$ over $100 \mathrm{~m}$. The transition from a higher viscosity has been introduced to promote uniform mixing and damp any spurious numerical instabilities at the open boundaries. The bottom friction was specified by a constant Manning's coefficient of $n=0.02 \mathrm{~s} \mathrm{~m}^{-1 / 3}$, which is close to what is typical in marine resource assessment applications (e.g. [18]).

\subsection{Sensitivity of vortex trajectory to inlet width $\left(W_{i}\right)$}

We first consider the square lagoon case $\left(L_{1}\right)$ where the domain sides are equidistant ( $L=W=5 \mathrm{~km}$ ). The focus is again on the half of the domain where the vortex of positive $\omega$ is propagating, on the grounds of symmetry.

The first criteria that should be observed is whether the dimensionless ratio $\left({ }^{W_{i}} / U T\right)$ accurately indicates the behavior of the vortices. According to the inlet width variation and the imposition of the same flux in all cases, the velocity at the inlet varies sinusoidally with a maximum of $\approx 0.6 \mathrm{~m} \mathrm{~s}^{-1}$, when $W_{\mathrm{i}}=0.75 \mathrm{~km}, 0.45 \mathrm{~m} \mathrm{~s}^{-1}$ for $W_{\mathrm{i}}=1 \mathrm{~km}$, and 0.36 $\mathrm{m} \mathrm{s}^{-1}$ for $W_{\mathrm{i}}=1.25 \mathrm{~km}$. The tidal period is $6 \mathrm{~h}$, so that the dimensionless parameter ${ }^{W_{\mathrm{i}}} / U T$ takes the value 0.161 for $W_{\mathrm{i}}=1.25 \mathrm{~km}, 0.103$ for $W_{\mathrm{i}}=1.00 \mathrm{~km}$, and 0.058 for the narrowest inlet. From theory and the previous results, the dipoles are expected to be flushed away with the ebb tide for the case with $W_{\mathrm{i}}=1.25 \mathrm{~km}$, but should otherwise remain in the lagoon as $W_{\mathrm{i}} / U T<0.13$.

This behavior can indeed be observed in Fig. 7. In the case of $W_{\mathrm{i}}=1.25 \mathrm{~km}$, changes in the vorticity field over time demonstrate how vortices do in fact dissipate as they return towards the inlet and get flushed within a single $T$. This is also shown by the tracking algorithm in Fig. 7c, where vortices cease to be traced until the subsequent flood tide that leads to the formation and propagation of a new recirculation zone. The vorticity intensity progressively increases as the width of the inlet is reduced, through an accelerated inlet velocity.

The results of Fig. 7 can be further complemented by the contour plots of the $\omega$ field that also indicate the trajectory of the vortex within the bottom half of the lagoon (Fig. 8). It should be remarked that in Fig. 8 the entire domain is included to demonstrate that despite the unstructured nature of the mesh, symmetry in terms of the vortices formed and the hydrodynamics is effectively maintained. The contour is plotted at $t \approx 3 T$ which corresponds to the start of an ebb tide, and also the point of maximum propagation of the dipoles that will eventually be flushed away $\left(W_{\mathrm{i}}=1.25 \mathrm{~km}\right.$, shown in blue). In the other cases, shown in red $\left(W_{\mathrm{i}}=1.00 \mathrm{~km}\right)$ and black $\left(W_{\mathrm{i}}=0.75 \mathrm{~km}\right)$, vortices from the preceding tidal cycles are still observed within the lagoon interior.

\subsection{Sensitivity of vortex trajectory to lagoon aspect ratio ( $L$ / W)}

Thetis predictions also demonstrate that the overall lagoon shape will influence the fate of internal vortices. For $L_{25 / 4}$, the longest domain, vortices tend to dissipate within a single period. This is shown by the drop in vorticity that happens at approximately $t=0.35 T$ after the identification of the vortex, which appears to be consistent for every case of $W_{\mathrm{i}}$ (Fig. 7a). Nonetheless, there are still differences observed for different inlet widths; a wider inflow distribution corresponds to an earlier drop in the vorticity (see Fig. 7a). Visualizing 


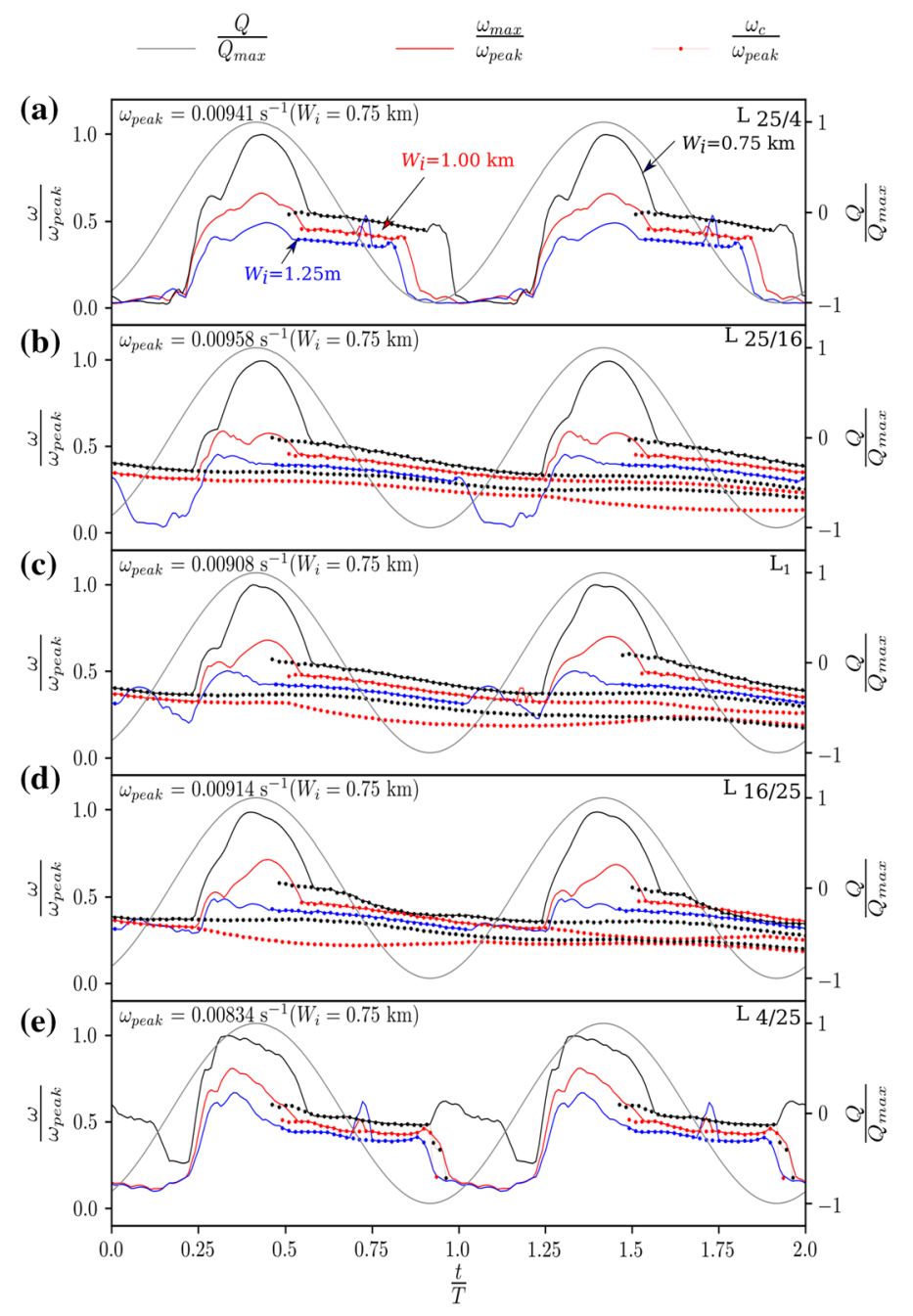

Fig. 7 Normalized maximum vorticity for the five lagoons of different $L / W$. For $W_{\mathrm{i}}=0.75 \mathrm{~km}$ (black lines), $W_{\mathrm{i}} / U T=0.058$, for $W_{\mathrm{i}}=1.00 \mathrm{~km}$ (red lines), $W_{\mathrm{i}} / U T=0.103$ and for $W_{\mathrm{i}}=1.25 \mathrm{~km}$ (blue lines), $W_{\mathrm{i}} / U T=0.161$. The lines with markers correspond to $\omega_{c}$ of traceable vortices in the confined lagoons. For $W_{\mathrm{i}}$ $=1.25 \mathrm{~km}$, regardless of $L / W$ value, vortices get dissipated within $1 T$

$\omega$ and the $\omega_{c}$ trajectory it becomes apparent that this dissipation is caused by the presence of the (no-slip) side walls close to the inlet (see the blue trajectory in Fig. 9).

Results for $L_{25 / 16}$ are plotted in Fig. 7b. Unlike $L_{25 / 4}$, vortices dissipate only when the inlet width is equal to $1.25 \mathrm{~km}$ indicating that their behavior is governed by the dimensionless parameter ${ }^{W_{\mathrm{i}}} /_{U T}$ similarly to the square case $L_{1}$, rather than the proximity of the side walls. Indeed for $W_{\mathrm{i}}=1.25 \mathrm{~km}$ the main vortex structures get flushed within 0.55 $T$. Moreover, vortices for $L_{25 / 16}$ tend to endure substantially longer than in all other $L / W$ configurations tested. Initially unhindered by side wall effects, the vortices propagate close to the centerline of the lagoon until they are redirected and start to dissipate through the 


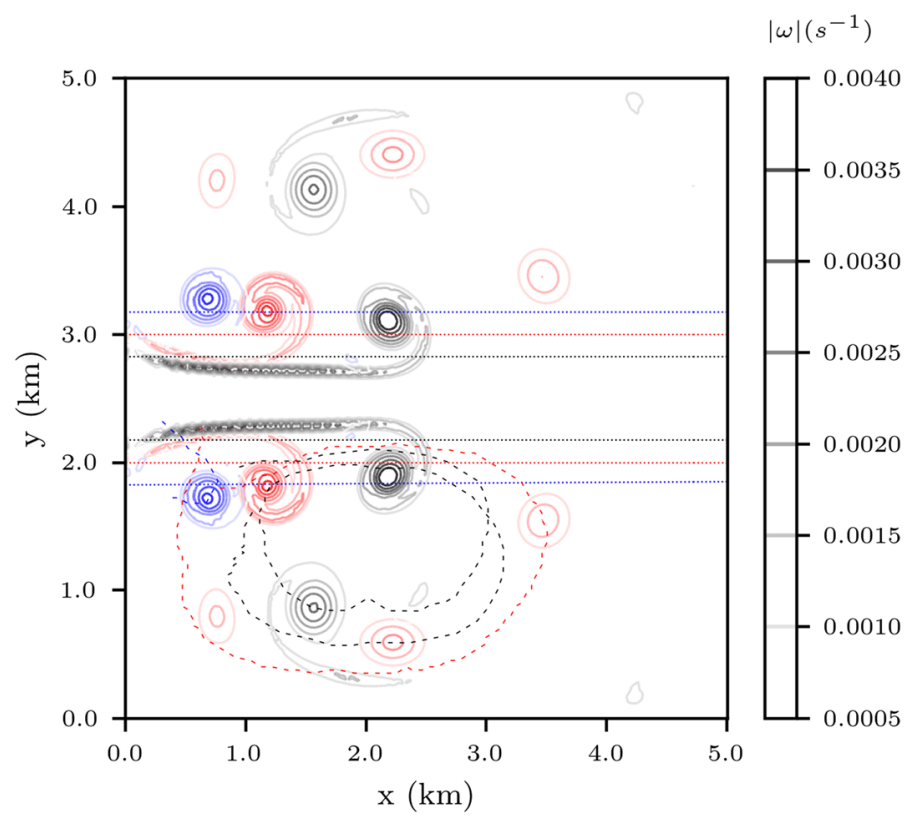

Fig. 8 Vorticity field at $t \approx 17.8 \mathrm{~h}$ (after ramp up) in lagoon $L_{1}$ with $W_{\mathrm{i}}=0.75 \mathrm{~km}$ (black), $W_{\mathrm{i}}=1.00 \mathrm{~km}$ (red) and $W_{\mathrm{i}}=1.25 \mathrm{~km}$ (blue). The same $\omega$ scale is used for all contours and individual cases are differentiated by the line color

Fig. 9 Trajectories of vortices for the five different aspect ratios with an inlet width of $W_{\mathrm{i}}=1.00 \mathrm{~km}$. The same scale is used for all contours and individual cases are differentiated by the line color

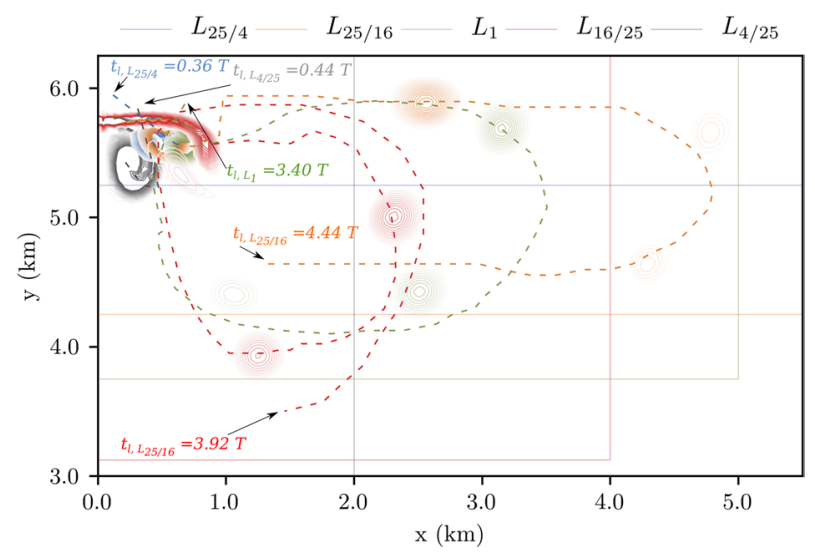

influence of the shallower bathymetry towards the coastal boundary (see the orange trajectory in Fig. 9).

The transient $\omega$ field for $L_{16 / 25}$ shows that vortices dissipate through a combination of sloping bathymetry and proximity of the inlet to the coastal side of the lagoon. This leads to further mixing, where the dipoles move back towards the inlet and even merge with the next pair of vortices (see the red trajectories in Fig. 9). Figure $7 \mathrm{~d}$ shows the intermediate $L_{16 / 25}$ case where for the 0.75 and $1.00 \mathrm{~km}$-wide inlets, trends observed in $L_{1}$ are closely followed. For $L_{4 / 25}$, vortices tend to remain closer to the inlet, a behavior attributed to the 
increased influence of the friction and reduced wave celerity in shallower waters, largely due to the effect of the sloping bathymetry that influences the entire lagoon. The flushing ratio $\left(W_{\mathrm{i}} / U T\right)$, the maximum $\omega_{\text {peak }}$ and the average lifetime of the vortices are summarized in Table 2 for completeness.

\subsection{Sensitivity of vortex trajectory to lagoon bathymetry}

Variations in bathymetry can be significant in coastal applications and must be acknowledged for their influence. In Table 2 an interesting pattern emerges with regard to the values of the vortex lifetime $t_{l}$ for $W_{\mathrm{i}}=1.00$ and $W_{\mathrm{i}}=0.75 \mathrm{~km}$. Even though the maximum peak vorticity is consistently encountered for $W_{\mathrm{i}}=0.75 \mathrm{~km}$, the associated vortices in $L_{25 / 16}, L_{1}$ and $L_{16 / 25}$ survive for less time than the $W_{\mathrm{i}}=1.00 \mathrm{~km}$ case. As a consequence of the greater momentum of the inflow associated with the narrower inlet (for $W_{\mathrm{i}}=0.75$ $\mathrm{km}$ ), vortices are advected closer to the shallower section of the lagoon and along the coast. Increased effects of friction become more pronounced and expedite the dissipation of vorticity, eventually contributing to a reduced lifetime $t_{l}$ relative to the $W_{\mathrm{i}}=1.00 \mathrm{~km}$ case.

We consider an additional case to demonstrate the implications of bathymetry on the fate of the vortices formed by the inlet flow. In this case the bathymetry of lagoon $L_{1}$ is represented by a gradient from $40 \mathrm{~m}$ at the inlet of the lagoon to $0 \mathrm{~m}$ at the coast; hence the sloping bathymetry covers $100 \%$ of the domain instead of $40 \%$ from previously. Results obtained with an inlet width of $0.75 \mathrm{~km}$ are compared against the original $L_{1}$ in Figs. 10 and 11 . As suggested by Table 2 in the original $L_{1}$, the vortex lifetime $t_{l}$ is approximately $3.13 \mathrm{~T}$. In the case of the $100 \%$ sloping bathymetry, this value reduces to $1.71 \mathrm{~T}$. The trajectory is also significantly altered, with the vortices encountering more resistance as they move closer to the coast.

Table 2 Comparison of results obtained for different lagoon aspect ratios $L / W$

\begin{tabular}{lllll}
\hline Lagoon & $W_{\mathrm{i}}(\mathrm{km})$ & $W_{\mathrm{i}} / U T(-)$ & $\omega_{\text {peak }} \times 10^{-3}\left(\mathrm{~s}^{-1}\right)$ & $t_{l}(T=6 \mathrm{~h})$ \\
\hline$L_{25 / 4}$ & 0.75 & 0.058 & 9.41 & $0.41 T$ \\
& 1.00 & 0.103 & 6.23 & $0.36 T$ \\
& 1.25 & 0.161 & 5.04 & $0.28 T$ \\
$L_{25 / 16}$ & 0.75 & 0.058 & 9.58 & $3.14 T$ \\
& 1.00 & 0.103 & 5.86 & $4.44 T$ \\
& 1.25 & 0.161 & 4.35 & $0.52 T$ \\
$L_{1}$ & 0.75 & 0.058 & 9.08 & $3.13 T$ \\
& 1.00 & 0.103 & 6.47 & $3.40 T$ \\
& 1.25 & 0.161 & 4.57 & $0.55 T$ \\
$L_{16 / 25}$ & 0.75 & 0.058 & 9.14 & $3.00 T$ \\
& 1.00 & 0.103 & 6.74 & $3.92 T$ \\
& 1.25 & 0.161 & 4.47 & $0.56 T$ \\
$L_{4 / 25}$ & 0.75 & 0.058 & 8.34 & $0.50 T$ \\
& 1.00 & 0.103 & 6.72 & $0.44 T$ \\
& 1.25 & 0.161 & 5.57 & $0.42 T$ \\
\hline
\end{tabular}

$\omega_{\text {peak }}$ is the maximum vorticity recorded in the bottom half of the monitoring domain, where the positive vortex was tracked. The predicted lifetime $t_{l}$ of persistent vortices generated at ebb tides is also included 


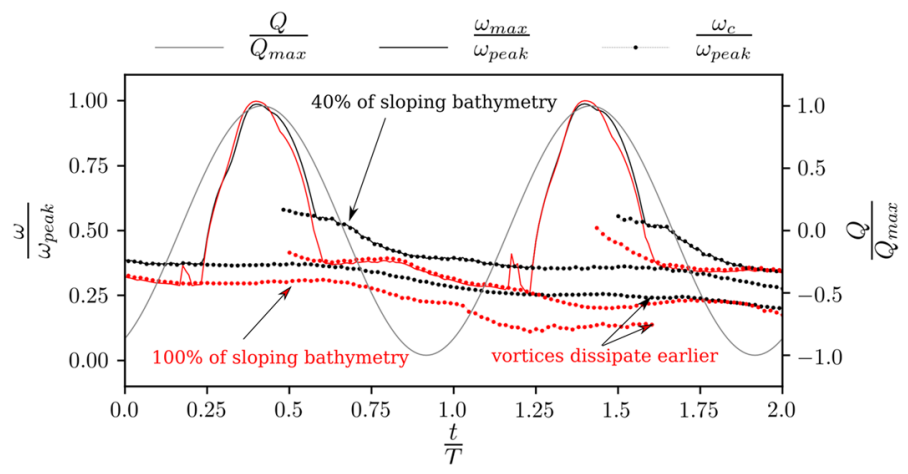

Fig. 10 Normalized vorticity for the $L_{1}$ lagoon geometry and $W_{\mathrm{i}}=0.75 \mathrm{~km}$, for cases with sloping bathymetry covering $40 \%$ and $100 \%$ of the domain

Fig. 11 Vortex trajectories for the $L_{1}$ lagoon geometry and $W_{\mathrm{i}}=$ $0.75 \mathrm{~km}$, for cases with sloping bathymetry covering $40 \%$ (black contours) and $100 \%$ (red contours) of the domain. The same $\omega$ scale is used for all contours and individual cases are differentiated by the line color

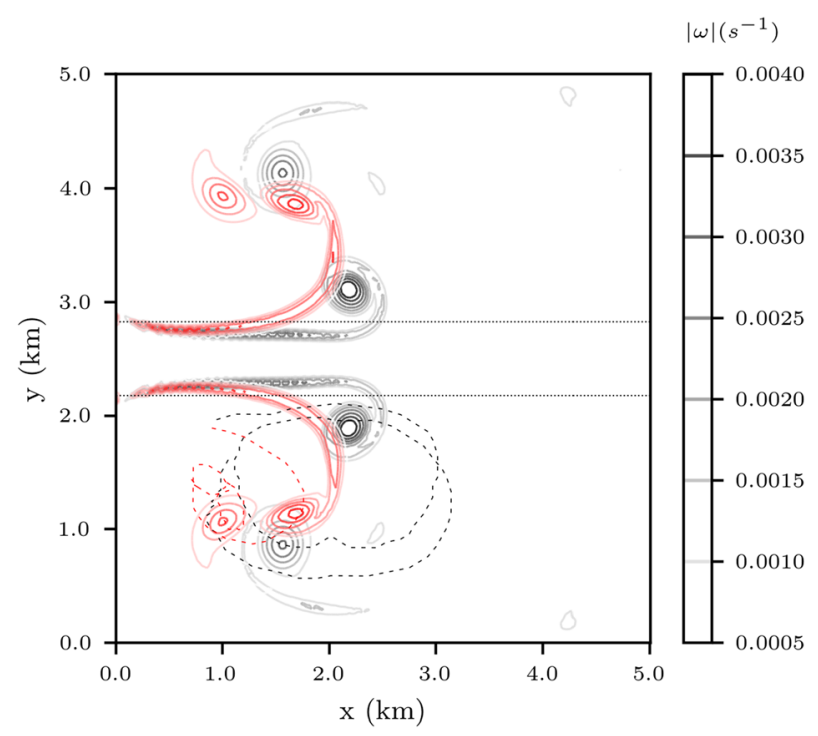

These predictions can be better understood by observing the bed shear stress $\left(\tau_{b}\right)$ parametrization in Eq. (4). As the depth $\left(H_{d}\right)$ reduces with the sloping bathymetry, the $\tau_{b}$ term that determines the energy dissipation rate increases. In turn, the depth-averaged velocity magnitude $|\mathbf{u}|$ also increases, contributing further to friction effects based again on the same Eq. (4). In addition, the sloping bathymetry leads to differences in $|\mathbf{u}|$ within the vortex. This leads to an uneven rotation rate that diverts the trajectory of the core depending on its clockwise/counter-clockwise rotation. This asymmetry with regard to the depth-averaged rotational velocities corresponds to vortex stretching effects in the propagation to shallower waters and an equivalent contraction as vortices are diverted to deeper waters. In Fig. 11, and consistently with Fig. 8, the entire domain is included illustrating how the trajectories of the counter-rotating vortices are diverted in opposing directions at an earlier stage of their evolution.

Consequently, the sloping bathymetry can have a significant impact on the dissipation and fate of the vortices. Indeed, both cases in Fig. 10 reach comparable peak vorticity 
values but in the case where the sloping bathymetry covers the entire domain, vorticity dissipates at an accelerated rate.

\subsection{Discussion}

The idealized lagoon representation considered in this work has highlighted certain parameters which influence the trajectory and lifetime of large-scale vortices formed by inlet water jets. Using the $\omega$ field and the $\left|\Gamma_{c}\right|$ quantity around the peaks it has been demonstrated how the trajectory and lifetime of vortices could be monitored over time. Beginning from the fundamental study of Wells and Heijst [13, 39], it was shown how the estuarine/lagoon geometry surrounding tidal inlets will play a significant role on the fate of vortices in practical cases. In the context of tidal range structure designs, several assumptions were made. For example, the distribution of the hydraulic structures does not have to be in a single location as was the case in all the scenarios considered here.

In fact, the operation of tidal power plants involves the coordination of turbines and sluice gates. These operate at different times of the tidal cycle and therefore may act to exacerbate or dissipate vortices within the lagoons depending on their configuration [23]. There are also a wide range of control sequences for regulating case-specific inflows associated with the power generation strategy. For example, the La Rance barrage in St Malo (France) features significant holding periods at ebb tide to facilitate a sufficient head difference between the inner and outer water levels with a view to generating power efficiently through bulb turbines as the tide recedes. However, these strategies could be altered in time subject to spring-neap tidal conditions [28] and this might lead to significantly variable flow patterns and internal vortex trajectories.

The inlet flow for our idealized cases was assumed to be sinusoidal. In practice, the flow will vary based upon the characteristics of the turbine and sluice gate structures as well as the transient tidal conditions. Improved numerical modeling should therefore feature appropriate parameterizations that reflect the technology installed in potential tidal range structure schemes. Regarding the ensuing effects of these vortices, the large-scale vortex structures predicted here will not necessarily have a noticeable impact on the power generation function of tidal (or more generally hydro-) power plants, as reported in depth-averaged model assessments [24]. The predictions are primarily of interest in terms of their influence on hydro-environmental processes such as sediment transport and scalar mixing. A better understanding of the links that arise between these hydrodynamic structures and water quality or siltation could shed light on the potential long-term environmental effects of such engineering developments.

Furthermore, bed geomorphology will lead to spatially varying bed friction, with irregular features such as shallow reefs and headlands (e.g. [9]) dictating local hydrodynamics and by extension the fate of vortices. Given, these assumptions, this paper has focused on presenting a method for determining the fate of vortices formed in close proximity to coastal and offshore developments. This methodology can now be adapted to the assessment of these structures once the practicalities raised in this discussion (which are certainly not exhaustive) are acknowledged using the appropriate numerical methods that have been developed to represent the operation of realistic tidal range structures in coastal and estuarine environments [11,24]. Going forward, it would be interesting to identify correlations between vortex fate, nearfield morphodynamics and ecological implications induced by the introduction of tidal inlets and coastal reservoirs. 


\section{Conclusions}

This paper has focused on the development, validation and application of numerical tools to identify vortex hydrodynamic structures associated with flows through tidal inlets and coastal reservoirs/lagoons. Such flow features can be encountered in a range of coastal and estuarine conditions; a particular application discussed here is the design of tidal range energy projects. Understanding the implications of long-enduring vortices adjacent to hydraulic structures has been identified as one of the hydrodynamic challenges that needs to be addressed in evaluating the environmental impacts of marine or hydropower infrastructure. Using two test cases, we have demonstrated that the coastal ocean modeling solver, Thetis, is an appropriate tool to simulate the flow through tidal inlets and tidal lagoon inflows in a manner that predicts the formation of large, jetinduced recirculation zones.

We discussed how the non-dimensional ratio ${ }^{W_{\mathrm{i}}} / U T$ can be a useful indicator as to whether vortices will propagate further into confined lagoons or be flushed as the oscillatory flow ebbs (or changes direction depending on the side of the tidal inlet we are interested in). However, through a set of idealized scenarios we highlight the significance of bed friction and bathymetry profile, and indicate how they can influence both the trajectory and the dissipation rate of the vortices.

A simulation methodology was presented that traces moving vortex structures by identifying peaks in the vorticity field $(\omega)$ and quantifying the circulation $\left(\left|\Gamma_{c}\right|\right)$ to assess whether these points in time correspond to a distinct vortex trajectory. These methods were used to consider the effect of several design parameters for idealized tidal lagoons or coastal reservoirs. For instance, the inlet width was shown to impact significantly upon the flushing and dissipation behavior of the vortices, with a wider distribution of the inlet flow leading to a greater $W_{\mathrm{i}} / U T$ value and an inflow of lower momentum that leads to an expedited dissipation of the primary vortex structures. Design characteristics such as the aspect ratio of the lagoons $(L / W)$ were also shown to impact the dissipation of vortices as well as the propagation trajectory over time. Rapid vortex dissipation was also promoted by the side walls or the proximity of the inlet to the coast, particularly for very large or small lagoon aspect ratios. Finally, it is proposed that the methodology for assessing the fate of vortices should be employed to model more realistic scenarios that consider further technical and operational aspects associated with the design of coastal structures, such as tidal range power generation schemes.

Acknowledgements The authors would like to thank Georgios Deskos and Dr. James R. Percival (Imperial College London) for frequent discussions that contributed to the formulation of the vortex tracking algorithm and the input of Prof. Graham Hughes (Imperial College London) on an earlier version of the manuscript. The authors would also like to thank Prof. Scott A. Socolofsky (Texas A\&M University) for the supplementary information regarding previous experimental campaigns of relevance to this work. Carolanne Vouriot acknowledges the support from the EPSRC Centre for Doctoral Training in Fluid Dynamics across Scales (EP/L016230/1). Athanasios Angeloudis acknowledges the support of the NERC fellowship Grant NE/R013209/1. Further support for this work stemmed from EPSRC under Grants EP/L000407/1 and EP/ M011054/1. The authors are also thankful for the insightful and constructive feedback of the anonymous reviewers that helped improve the manuscript.

Open Access This article is distributed under the terms of the Creative Commons Attribution 4.0 International License (http://creativecommons.org/licenses/by/4.0/), which permits unrestricted use, distribution, and reproduction in any medium, provided you give appropriate credit to the original author(s) and the source, provide a link to the Creative Commons license, and indicate if changes were made. 


\section{References}

1. Stansby P, Chini N, Lloyd P (2016) Oscillatory flows around a headland by 3D modelling with hydrostatic pressure and implicit bed shear stress comparing with experiment and depth-averaged modelling. Coast Eng 116:1-14. https://doi.org/10.1016/j.coastaleng.2016.05.008

2. Callendar W, Klymak JM, Foreman MGG (2011) Tidal generation of large sub-mesoscale eddy dipoles. Ocean Sci 7(4):487-502. https://doi.org/10.5194/os-7-487-2011

3. Falconer RA, Wolanski E, Mardapitta-Hadjipandeli L (1986) Modeling tidal circulation in an island's wake. J Waterw Port Coast Ocean Eng 112(2):234-254. https://doi.org/10.1061/ (ASCE)0733-950X(1986)112:2(234)

4. Neill SP, Jordan JR, Couch SJ (2012) Impact of tidal energy converter (TEC) arrays on the dynamics of headland sand banks. Renew Energy 37(1):387-397. https://doi.org/10.1016/j.renen e.2011.07.003

5. Perez-Ortiz A, Borthwick AGL, McNaughton J, Avdis A (2017) Characterization of the tidal resource in Rathlin Sound. Renew Energy 114:229-243. https://doi.org/10.1016/j.renen e.2017.04.026

6. Yuan D, Lin B, Falconer RA (2007) A modelling study of residence time in a macro-tidal estuary. Estuar Coast Shelf Sci 71(3):401-411. https://doi.org/10.1016/j.ecss.2006.08.023

7. Falconer RA, Lin B (1997) Three-dimensional modelling of water quality in the Humber Estuary. Water Res 31(5):1092-1102. https://doi.org/10.1016/S0043-1354(96)00333-8

8. Wolanski E (1988) Tidal jets, nutrient upwelling and their influence on the productivity of the alga Halimeda in the Ribbon Reefs, Great Barrier Reef. Estuar Coast Shelf Sci 26(2):169-201

9. Kim JW, Ha HK, Woo SB (2017) Dynamics of sediment disturbance by periodic artificial discharges from the world's largest tidal power plant. Estuar Coast Shelf Sci 190:69-79

10. Hendry C (2017) The role of tidal lagoons-final report, Technical Report, UK Government. https:// hendryreview.files.wordpress.com/2016/08/hendry-review-final-report-english-version.pdf

11. Cornett A, Cousineau J, Nistor I (2013) Assessment of hydrodynamic Impacts from tidal power lagoons in the Bay of Fundy. Int J Mar Energy 1:33-54

12. Falconer RA, Angeloudis A, Ahmadian R (2018) Modeling hydro-environmental impacts of tidal range renewable energy projects in coastal waters. Handbook of Coastal and Ocean Engineering, Chapter 55. World Scientific, Singapore, pp 1553-1574. https://doi.org/10.1142/9789813204027_0055

13. Wells MG, van Heijst GF (2003) A model of tidal flushing of an estuary by dipole formation. Dyn Atmos Oceans 37(3):223-244

14. Kim D, Lynett PJ, Socolofsky SA (2009) A depth-integrated model for weakly dispersive, turbulent, and rotational fluid flows. Ocean Model 27(3):198-214

15. Whilden KA (2015) Investigation of tidal exchange and the formation of tidal vortices at Aransas Pass, Texas, USA. PhD thesis. Texas A\&M University

16. Bryant DB, Whilden KA, Socolofsky SA, Chang K-A (2012) Formation of tidal starting-jet vortices through idealized barotropic inlets with finite length. Environ Fluid Mech 12(4):301-319. https://doi. org/10.1007/s10652-012-9237-4

17. Lewis MJ, Angeloudis A, Robins PE, Evans PS, Neill SP (2017) Influence of storm surge on tidal range energy. Energy 122:25-36. https://doi.org/10.1016/j.energy.2017.01.068

18. Angeloudis A, Piggott MD, Kramer SC, Avdis A, Coles D (2017) Comparison of 0-D , 1-D and 2-D model capabilities for tidal range energy resource assessments. In: Proceedings of the EWTEC 2017 conference. Cork, pp 1-10. https://doi.org/10.17605/OSF.IO/RYT6H

19. Angeloudis A, Falconer RA (2017) Sensitivity of tidal lagoon and barrage hydrodynamic impacts and energy outputs to operational characteristics. Ren Energy 114(A):337-351. https://doi.org/10.1016/j. renene.2016.08.033

20. Wolf J, Walkington IA, Holt J, Burrows R (2009) Environmental impacts of tidal power schemes. Proc ICE Mar Eng 162(4):165-177. https://doi.org/10.1680/maen.2009.162.4.165

21. Burrows R, Walkington IA, Yates NC, Hedges TS, Wolf J, Holt J (2009) The tidal range energy potential of the West Coast of the United Kingdom. Appl Ocean Res 31(4):229-238. https://doi. org/10.1016/j.apor.2009.10.002

22. Jeffcoate P, Stansby P, Apsley D (2016) Flow and bed-shear magnification downstream of a barrage with swirl generated in ducts by stators and rotors. J Hydraul Eng 143(2):06016023. https://doi. org/10.1061/(ASCE)HY.1943-7900.0001228

23. Angeloudis A, Ahmadian R, Falconer RA, Bockelmann-Evans B (2016) Numerical model simulations for optimisation of tidal lagoon schemes. Appl Energy 165:522-536. https://doi.org/10.1016/j.apene rgy.2015.12.079 
24. Angeloudis A, Falconer RA, Bray S, Ahmadian R (2016) Representation and operation of tidal energy impoundments in a coastal hydrodynamic model. Renew Energy 99:1103-1115. https://doi. org/10.1016/j.renene.2016.08.004

25. Karna T et al (2016) Thetis. https://github.com/thetisproject/thetis/. Accessed 07 Aug 2017

26. Rathgeber F, Ham DA, Mitchell L, Lange M, Luporini F, McRae ATT, Bercea G-T, Markall GR, Kelly PHJ (2016) Firedrake: automating the finite element method by composing abstractions. ACM Trans Math Softw 43(3):24:1-24:27. https://doi.org/10.1145/2998441

27. Kärnä T, Kramer SC, Mitchell L, Ham DA, Piggott MD, Baptista AM (2018) Thetis coastal ocean model: discontinuous Galerkin discretization for the three-dimensional hydrostatic equations. Geosci Model Dev Discuss 2018:1-36. https://doi.org/10.5194/gmd-2017-292

28. Angeloudis A, Kramer SC, Avdis A, Piggott MD (2018) Optimising tidal range power plant operation. Appl Energy 212:680-690. https://doi.org/10.1016/j.apenergy.2017.12.052

29. Falconer R, Owens PH (1987) Numerical simulation of flooding and drying in a depth-averaged tidal flow model. Inst Civ Eng Proc Part 2 2(83):161-80

30. Kärnä T (2011) A fully implicit wetting-drying method for DG-FEM shallow water models, with an application to the Scheldt Estuary. Comput Methods Appl Mech Eng 200(5):509-524

31. Balay S, Abhyankar S, Adams MF, Brown J, Brune P, Buschelman K, Dalcin L, Eijkhout V, Gropp WD, Kaushik D, Knepley MG, McInnes LC, Rupp K, Smith BF, Zampini S, Zhang H, Zhang H (2016) PETSc users manual. Technical report ANL-95/11-Revision 3.7. Argonne National Laboratory

32. Neill SP, Angeloudis A, Robins PE, Walkington I, Ward SL, Masters I, Lewis MJ, Piano M, Avdis A, Piggott MD, Aggidis G, Evans P, Adcock TAA, Zidonis A, Ahmadian R, Falconer R (2018) Tidal range energy resource and optimization past perspectives and future challenges. Renew Energy. https:// doi.org/10.1016/j.renene.2018.05.007

33. Martin-Short R, Hill J, Kramer SC, Avdis A, Allison PA, Piggott MD (2015) Tidal resource extraction in the Pentland Firth, UK: potential impacts on flow regime and sediment transport in the Inner Sound of Stroma. Renew Energy 76:596-607. https://doi.org/10.1016/j.renene.2014.11.079

34. Jeffcoate P, Stansby P, Apsley D (2012) Flow due to multiple jets downstream of a barrage: experiments, 3-D computational fluid dynamics, and depth-averaged modeling. J Hydraul Eng 139(7):754-762

35. Ead SA, Rajaratnam N (2002) Plane turbulent wall jets in shallow tailwater. J Eng Mech 128(2):143-155

36. Geyer WR (1993) Three dimensional tidal flow around headlands. J Geophys Res Oceans 98(C1):955966. https://doi.org/10.1029/92JC02270

37. Deleersnijder E, Norro A, Wolanski E (1992) A three-dimensional model of the water circulation around an island in shallow water. Cont Shelf Res 12(7):891-906. https://doi.org/10.1016/02784343(92)90050-T

38. Signell RP, Geyer WR (1991) Transient eddy formation around headlands. J Geophys Res Oceans 96(C2):2561-2575. https://doi.org/10.1029/90JC02029

39. Wells MG, van Heijst GJF (2004) Dipole formation by tidal flow in a channel. In: International symposium on shallow flows. Balkema Publishers, Delft, pp 63-70

40. Kadiri M, Ahmadian R, Bockelmann-Evans B, Rauen W, Falconer R (2012) A review of the potential water quality impacts of tidal renewable energy systems. Renew Sustain Energy Rev 16(1):329-341. https://doi.org/10.1016/j.rser.2011.07.160 